\title{
A case report of advanced hepatocellular carcinoma treated with hepatic arterial infusion chemotherapy and sorafenib combination therapy followed by metastasectomy of lung and muscle metastases
}

\author{
Sang Yi Moon', Sang Young Han², Yang-Hyun Baek' \\ Department of Internal Medicine, 'Dong-A University College of Medicine, ${ }^{2}$ On Hospital, Busan, Korea
}

Received Nov. 17, 2021

Revised Dec. 8, 2021

Accepted Dec. 12, 2021
Currently, various tyrosine kinase inhibitors and immune checkpoint inhibitors have been suggested in the treatment guidelines for advanced hepatocellular carcinoma (HCC). However, sorafenib was the only systemic drug approved 10 years ago. In 2010, a woman diagnosed with $\mathrm{HCC}$ rupture and multiple lung metastases visited our hospital. At the time of visiting our hospital, she had undergone transarterial chemoembolization at another hospital to control bleeding due to HCC rupture. We treated her with hepatic arterial infusion chemotherapy and sorafenib combination therapy to increase the control of intrahepatic tumors in consideration of the modest efficacy of sorafenib. The intrahepatic tumor was almost controlled. Metastasectomy was performed to control lung oligometastasis. Subsequently, additional muscle metastasis was confirmed, and metastasectomy was performed. Although this is a very rare case, it shows that a multidisciplinary approach can improve the prognosis of patients with HCC. (J Liver Cancer 2022;22:57-62)

Keywords: Hepatocellular carcinoma; Intraarterial infusion; Metastasectomy; Case report

\section{INTRODUCTION}

Hepatocellular carcinoma (HCC) is the fourth most common cause of cancer-related deaths worldwide. ${ }^{1}$ Treatment options for HCC depend on the tumor stage and the patient's performance. ${ }^{2}$ According to the mainstream HCC guidelines, systemic therapy is recommended as a standard treatment for advanced HCC with extrahepatic metastasis. However, systemic therapy cannot completely eliminate these lesions. In addition, the low percentage of patients who exhibit pro-

\section{Corresponding author: Yang-Hyun Baek}

Department of Internal Medicine, Dong-A University College of Medicine, 32 Daesingongwon-ro, Seo-gu, Busan 49201, Korea

Tel. +82-51-240-2728, Fax. +82-51-240-2087

E-mail:p100100@dau.ac.kr longed survival is unsatisfactory.

Most patients with advanced HCC expire from hepatic failure or progression of intrahepatic lesions. Therefore, for advanced HCC with extrahepatic metastasis, an attempt has been made to manage extrahepatic lesions with locoregional therapy on the premise of appropriate intrahepatic lesion management; this was effective in some reports. ${ }^{3,4}$

In this report, we present a case in which hepatic arterial infusion chemotherapy (HAIC) and sorafenib combination therapy was administered for advanced HCC with lung metastasis. A complete response was maintained after metastasectomy for lung metastasis and newly developed muscle metastasis. This case report was described according to the CARE guidelines available from https://www.care-statement.org/. 


\section{CASE REPORT}

A 35-year-old woman visited another hospital in August 2010 due to sudden abdominal pain accompanied by distension. She was diagnosed with multiple HCCs with rupture (Fig. 1A, B). She underwent emergency transarterial chemoembolization (TACE) and visited our hospital in September 2010 for further treatment. She was known to be a carrier of hepatitis B. However, she did not undergo regular screening. There was no family history of note.

Initial laboratory findings were as follows: white blood cell count, 17,210/ $\mathrm{LL}$; hemoglobin, $9.6 \mathrm{~g} / \mathrm{dL}$; platelet count, 281,000/ $\mu \mathrm{L}$; total bilirubin, $1.4 \mathrm{mg} / \mathrm{dL}$; albumin, $4.4 \mathrm{~g} / \mathrm{dL}$; prothrombin time-international normalized ratio, 1.10; aspartate aminotransferase, $50 \mathrm{U} / \mathrm{L}$; alanine aminotransferase, $28 \mathrm{U} / \mathrm{L}$; hepatitis B e-antigen, positive; anti-hepatitis B e-antibody, negative; HBV DNA, 955 IU/mL; alpha-fetoprotein (AFP), >35,000 ng/mL; and protein induced by the absence of vitamin K or antagonist-II (PIVKA-II), 117 mAU/mL.

Initial chest computed tomography $(\mathrm{CT})$ revealed multiple lung metastases (Fig. 1C, D). On the liver dynamic CT performed 2 weeks after TACE, multiple HCCs showed a decrease in size and central necrosis compared to the initial CT, but multiple viable tumors were still observed in both lobes of the liver. Liver function was preserved with a Child-Pugh score of 5. The Eastern Cooperative Oncology Group performance status score was 0 . The HCC was classified as an advanced stage by the Barcelona Clinic Liver Cancer (BCLC) system and as stage IVB (T4N0M1) by the modified Union for International Cancer Control TNM classification.

We started entecavir ( $0.5 \mathrm{mg}$ once daily) for the treatment of hepatitis B. Sorafenib (400 mg twice daily) was administered, and HAIC combination therapy was performed considering the modest effect of sorafenib. HAIC using floxuridine (FUDR) at $0.3 \mathrm{mg} / \mathrm{kg}$ per day was administered for 14 days followed by withdrawal for 14 days, representing one cycle. After three cycles of HAIC and daily administration of sorafenib for 3 months, a follow-up CT showed a decrease in the proportion of viable tumors exhibiting enhancement in the liver and decreased size and the number of lung metastases. Serum AFP level declined from $>35,000 \mathrm{ng} / \mathrm{mL}$ to 6,581 $\mathrm{ng} / \mathrm{mL}$ and serum PIVKA-II level declined from $117 \mathrm{mAU} / \mathrm{mL}$ to $20 \mathrm{mAU} / \mathrm{mL}$. Consequently, combination therapy was continued.

After maintaining HAIC for 15 cycles with daily administration of sorafenib until February 2012, no further viable intrahepatic lesions were observed on CT (Fig. 2A, B). Chest CT showed three nodules in both lower lobes. However, the left nodule was too small to identify definite viability (Fig. 2C-E). Positron emission tomography computed tomography (PET-CT) was done to confirm the absence of viable portions, and biopsy was performed on the suspected lesions. However, only necrotic changes with fibrous tissue were identified (Fig. 2F). Since we judged that there were no viable tumors except for small lung metastases, we decided to stop combination therapy of HAIC with sorafenib and performed a metastasectomy for two nodules in the right lower lobe. Only one of the two nodules was identified as a metastatic tumor.

Tumor markers and imaging studies were followed up every 3 months following termination of combination therapy. In October 2013, the patient's serum AFP level elevated to
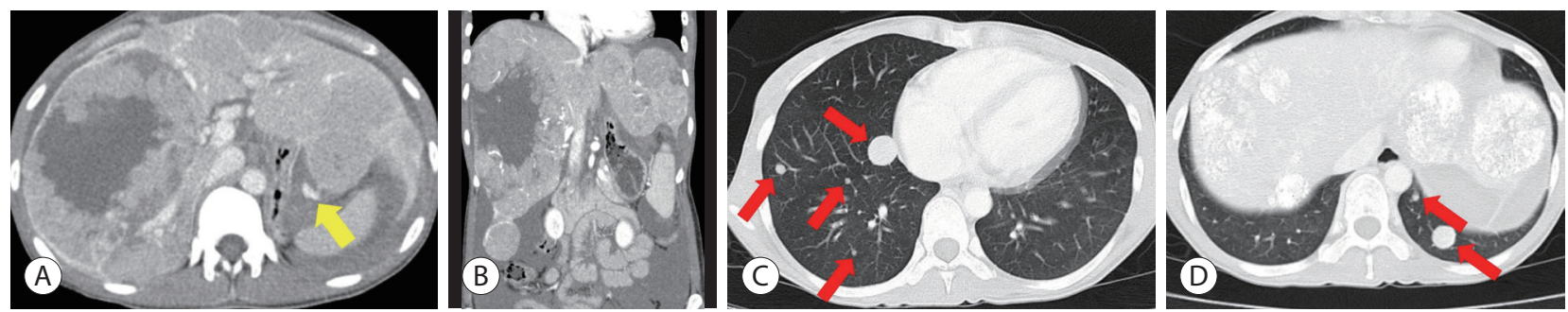

Figure 1. Computed tomography (CT) at the first diagnosis of hepatocellular carcinoma in August 2010. Abdominal CT showed multiple hypervascular masses in both lobes, extravasation of contrast media from the left lobe (yellow arrow), and hemoperitoneum in the arterial phase $(A, B)$. Chest $C T$ showed multiple lung metastases (red arrows) $(C, D)$. 
$340 \mathrm{ng} / \mathrm{mL}$ from $29.37 \mathrm{ng} / \mathrm{mL}$. No other changes were observed, except for a small nodule in the left lung. Therefore, we decided to perform a second metastasectomy, and the lesion was identified as a metastatic tumor. Her AFP level declined to normal level after the metastasectomy.

However, the patient's AFP levels began to rise again in June 2015. Liver magnetic resonance imaging (MRI), hepatic arterial angiography, and PET-CT were performed to identify a lesion. There was no evidence of recurrence. Meanwhile, in October 2016, the serum AFP level was elevated to 43,256 $\mathrm{ng} / \mathrm{mL}$. Recurrence of HCC, $25 \mathrm{~mm}$ in size, was observed in the S5/6 lesion on liver dynamic CT (Fig. 3). Laparoscopic hepatic wedge resection was performed considering the patient's liver function and the location of the recurrent HCC. Despite successful operation, the serum AFP level rose to $45,985 \mathrm{ng} / \mathrm{mL}$, which was higher than that before resection. While investigating the cause of the elevation in tumor markers, the patient reported that she had felt a mass on her right
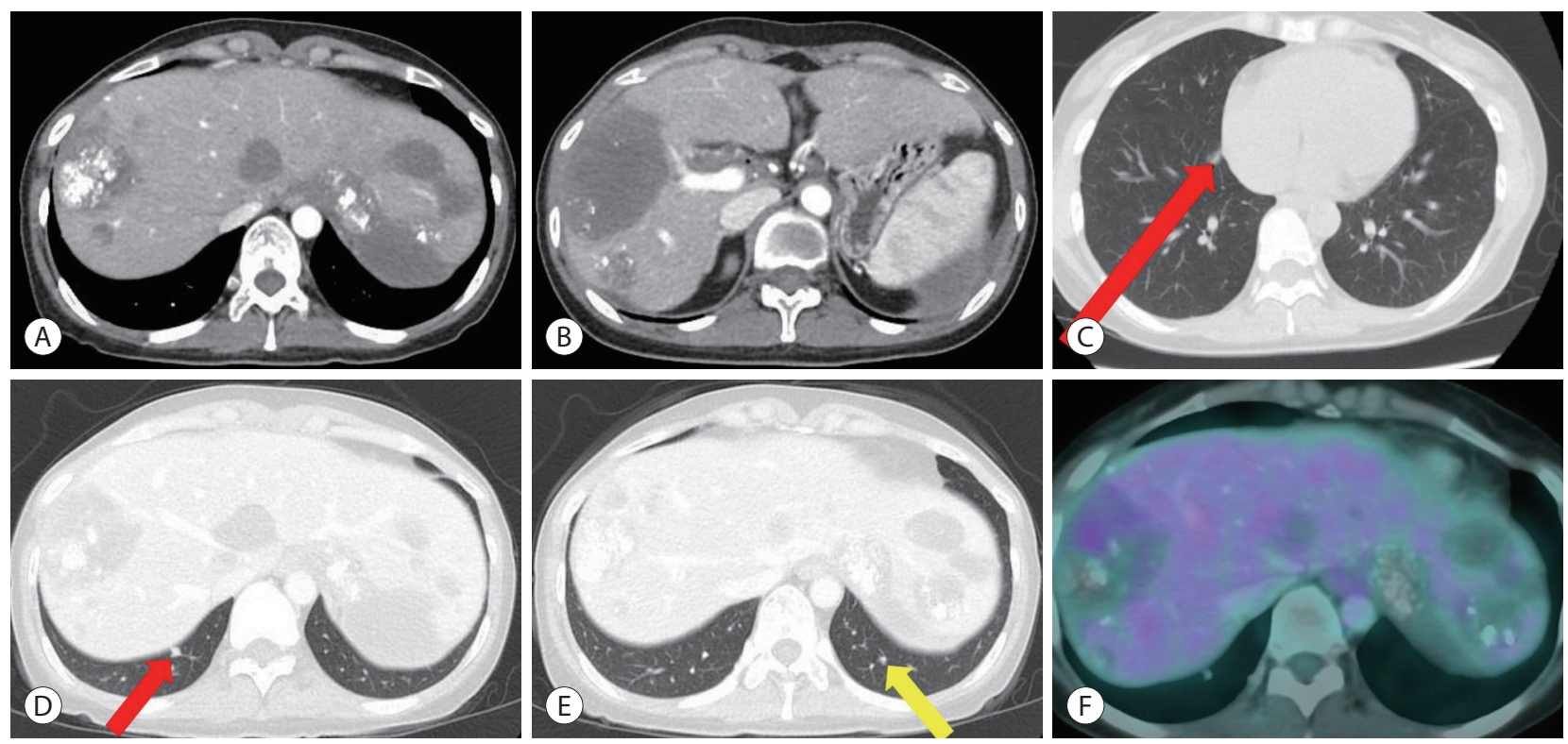

Figure 2. Computed tomography (CT) followed by 15 cycles of hepatic artery infusion chemotherapy and daily sorafenib administration in February 2012. Abdominal CT showed no viable tumor exhibiting enhancement in the liver in the arterial phase (A, B). Chest CT showed three nodules in both lower lobes. The left nodule (yellow arrow) was too small to identify definite viability. Thus, metastasectomy was only performed for the two nodules (red arrow) in the right lower lobe (C-E). Positron emission tomography CT showed a mild hypermetabolism in the S8 lesion that had been previously treated with transarterial chemoembolization, but only necrotic change with fibrous tissue was identified by biopsy (F).
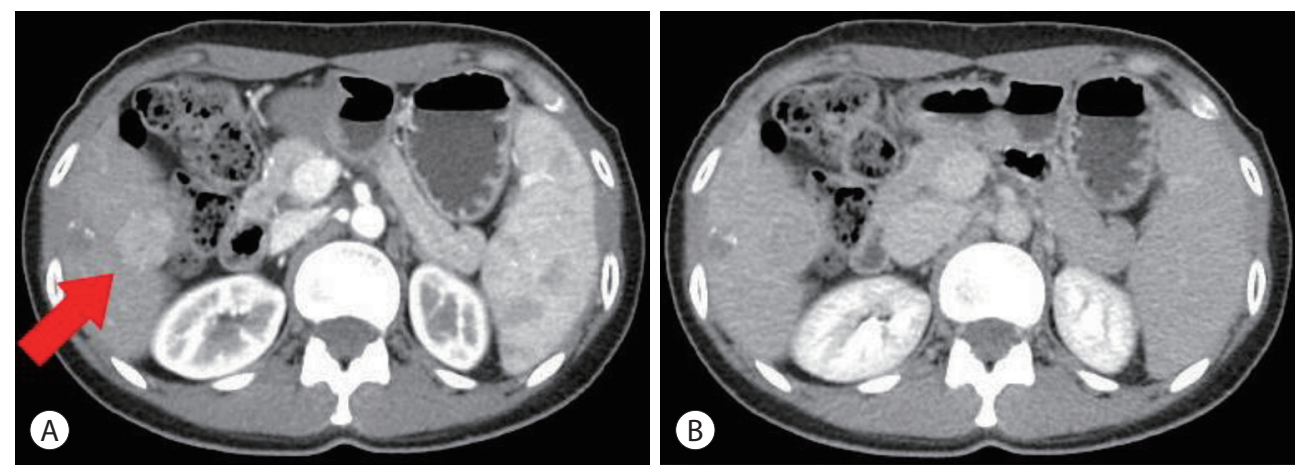

Figure 3. Abdominal computed tomography in October 2016, showing recurred hepatocellular carcinoma with a size of $25 \mathrm{~mm}$ and contrast enhancement in the arterial phase (red arrow) (A). Washout in the delayed phase (B) was found in the S5/6 lesion. 
thigh since 1 year ago. When performing thigh MRI, a mass of $5 \mathrm{~cm}$ in size was observed in the right semimembranous muscle, which showed weaker high signal intensity compared to the surrounding tissue in the T1-weighted image. It showed high signal intensity with inhomogeneity in the T2weighted image (Fig. 4A). In January 2017, a third metastasectomy, including some normal muscle tissues, was performed. The well-defined mass was not connected to the surrounding semimembranous muscle. The patient was histologically diagnosed with metastatic HCC (Fig. 4B). Following the third metastasectomy for muscle metastasis, serum AFP declined to $3,212 \mathrm{ng} / \mathrm{mL}$ after 3 months and normalized to $2.4 \mathrm{ng} / \mathrm{mL}$ after 6 months. There was no recurrence of HCC in the imaging study, including thigh MRI. Based on the image study and tumor marker normalization, we decided not to administer additional systemic chemotherapy. The patient was followed up until August 2021 in the outpatient clinic, showing no recurrence of HCC (Fig. 5).

\section{DISCUSSION}

According to data obtained from the Korean Nationwide Cancer Registry, 39.4\% of patients diagnosed with liver cancer for the first time have advanced HCC with BCLC stage C. ${ }^{5}$ Recently, with the development of new agents, combination treatment with tyrosine kinase inhibitors and immune checkpoint inhibitors has been recommended as the firstline treatment for advanced HCC. However, sorafenib was the only systemic drug approved 10 years ago. The efficacy of sorafenib has been shown to be modest, with an actual survival benefit of less than 3 months, tumor response rate of less than $5 \%$, and median survival time of less than 1 year. ${ }^{6}$ Therefore, various treatment methods have been tried. One of them is the combination therapy of HAIC and sorafenib. The combination of HAIC and sorafenib might have complementary effects on advanced HCC, with sorafenib prolonging survival through disease stabilization and HAIC shrinking tumors. In a phase I/II study comparing HAIC and sorafenib combination therapy group with sorafenib monotherapy group in advanced HCC in Japan, all responses including overall survival (OS) were improved. ${ }^{7}$ However, in a phase III randomized controlled trial, there were significant differences in progression-free survival and objective response rate, but no significant difference in OS. ${ }^{8}$ Interestingly, when the main portal vein invasion group was analyzed separately, the OS of the combination therapy group was 11.4 months and the OS of the sorafenib-alone group was 6.5 months, showing a significant difference $(P=0.05){ }^{8} \mathrm{Al}-$ though definite evidence for OS improvement is still lacking, there is still hope for good outcomes from combination therapy in advanced HCC.

HAIC can deliver chemotherapeutic agents directly to the hepatic artery that feeds HCC, enabling the infusion of high concentrations of chemotherapeutic agents into the tumor, thereby increasing the antitumor effect. The first-pass effect in the liver can minimize systemic toxicity by generating rela-
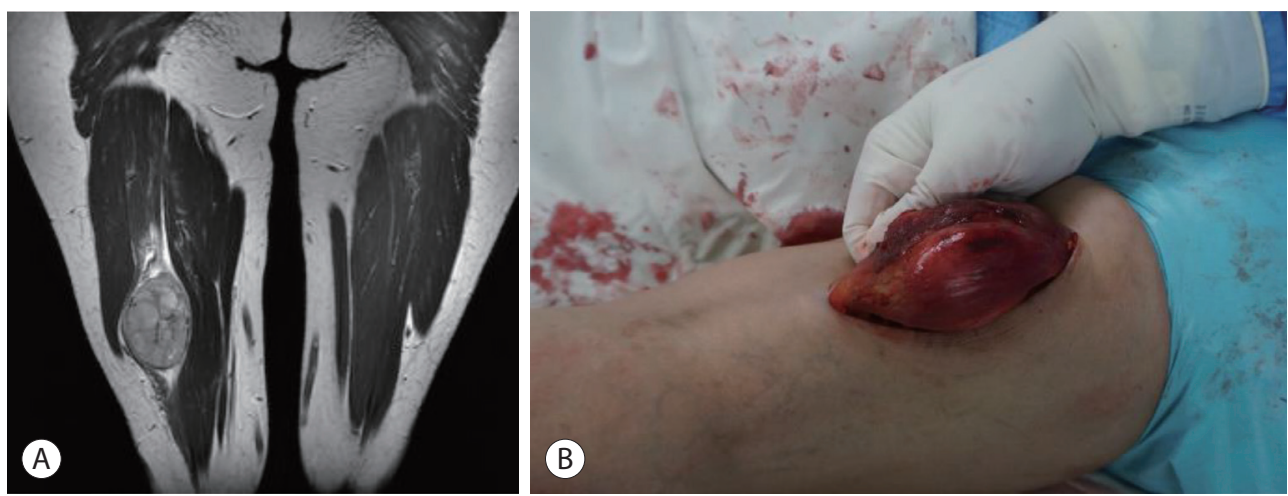

Figure 4. A thigh magnetic resonance imaging (MRI) was performed because a mass was felt on the right thigh from 1 year ago. T2-weighted MRI showed a heterogeneous signal intense mass, which was not observed to communicate with other muscles or soft tissues (A). Muscle metastasis taken before resection in the operating room (B). 
tively low systemic levels of chemotherapeutic agents. Various combinations of cisplatin, 5-fluorouracil (5-FU), and interferon have been reported as chemotherapeutic regimens for HAIC. The combination of cisplatin and 5-FU is the most commonly used therapy. ${ }^{9}$ Among the various regimens, FUDR was determined as the therapy for use in HAIC. FUDR, instead of 5-FU, was selected as the chemotherapeutic agent in HAIC because intra-arterial FUDR, a metabolite of fluorouracil, showed increased hepatic extraction (>95\%) and more than 10 -fold intrahepatic concentration. ${ }^{10}$ We assumed that in the present case, the combination treatment of sorafenib with HAIC further increased the tumor response and enabled the next treatment modality.

Extrahepatic metastasis is a well-known poor prognostic factor for patients' OS as shown in a previous study. ${ }^{11} \mathrm{Al}-$ though metastasectomy has a proven survival advantage for certain malignancies, the role of extrahepatic metastasectomy for advanced HCC is still under debate. In one retrospective study, when comparing the median survival of patients with advanced HCC with extrahepatic metastasis, extrahepatic metastasectomy was more effective than sorafenib monotherapy. ${ }^{12}$ More studies are needed to determine which group of patients with advanced HCC with extrahepatic metastasis will be effective for metastasectomy. In our case, after adequate control of the intrahepatic lesions, metastasectomy was performed to cure. Among extrahepatic metastases, skeletal muscle metastasis is very rare, despite an abundant blood supply. This rarity is presumably due to the contractility of the skeletal muscle, the local acidic environment due to the accumulation of lactic acid, and the presence of tumor suppressors or lymphocytes that can create an environment unfavorable for metastasis. ${ }^{13}$ It should be noted that although our case reported cure after metastasectomy of semimembranous muscle metastasis, muscle metastasis has a very poor prognosis.

In conclusion, we present a case in which a combination therapy of HAIC and sorafenib was administered for treatment of advanced HCC with lung metastasis. A complete response was maintained after metastasectomy for lung metastasis and newly developed muscle metastasis. The therapeutic effects of sorafenib and HAIC combination therapy have not been proven. When sorafenib was first introduced into Korea, it was decided to combine HAIC with the expectation of a better effect. Increased tumor response through sorafenib and HAIC combination therapy might have provided the patient with an opportunity for metastasectomy. Determining the treatment of advanced HCC with extrahepatic metastasis by the BCLC stage may limit the application of various treatments to individual patients. To determine whether a multidisciplinary approach would be helpful, patient's liver func-

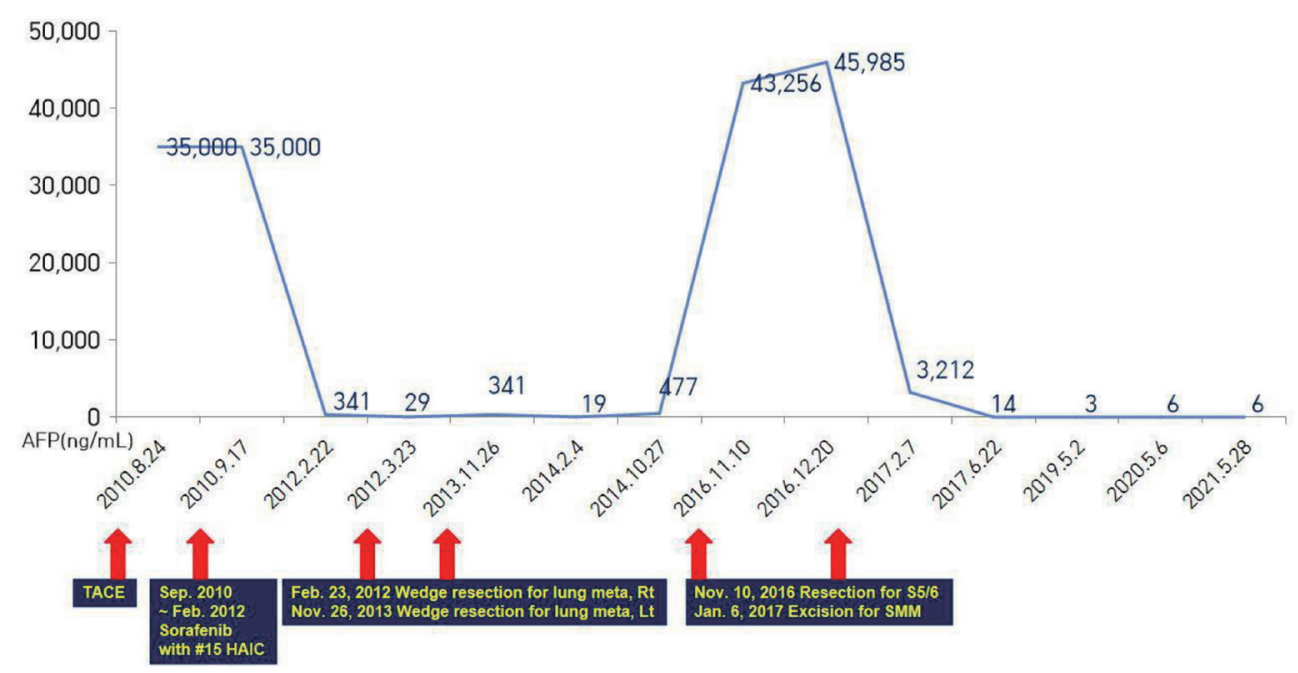

Figure 5. Summary of treatment course and changes in serum alpha-fetoprotein (AFP) levels. HAIC, hepatic arterial infusion chemotherapy; Rt., right; Lt., left; SMM, semimembranous muscle. 
tion, the extent of extrahepatic metastasis, presence of portal vein invasion, and control of intrahepatic lesions should be considered.

\section{Conflicts of Interest}

The authors have no conflicts of interest to disclose.

\section{Ethics Statement}

The present case report was approved by the Institutional Review Board (IRB) of the Dong-A University College of Medicine, Busan, Korea (IRB number: DAUHIRB-21-195). Informed consent was obtained from the patient. The study protocol conformed to the ethical guidelines of the Declaration of Helsinki of the World Medical Association.

\section{Funding Statement}

No funding to declare.

\section{Data Availability}

Data sharing not applicable to this article as no datasets were generated or analyzed for this case report.

\section{ORCID}

$$
\begin{array}{ll}
\text { Sang Yi Moon } & \text { https://orcid.org/0000-0002-8929-9250 } \\
\text { Sang Young Han } & \text { https://orcid.org/0000-0002-0485-9555 } \\
\text { Yang-Hyun Baek } & \text { https://orcid.org/0000-0002-9073-9233 }
\end{array}
$$

\section{Author Contribution}

Conceptualization: SYH, YHB

Data curation: $\mathrm{SYH}, \mathrm{YHB}$

Methodology: SYH, YHB

Project administration: SYH, YHB

Writing original draft: SYM

Writing review \& editing: YHB

Approval of final manuscript: all authors

\section{References}

1. Yang JD, Hainaut $P$, Gores GJ, Amadou A, Plymoth A, Roberts LR.
A global view of hepatocellular carcinoma: trends, risk, prevention and management. Nat Rev Gastroenterol Hepatol 2019;16:589604.

2. Llovet JM, Kelley RK, Villanueva A, Singal AG, Pikarsky E, Roayaie S, et al. Hepatocellular carcinoma. Nat Rev Dis Primers 2021;7:6.

3. Midorikawa Y, Takayama T, Nakayama H, Moriguchi M, Aramaki 0 , Yamazaki $S$, et al. Favorable outcomes of surgical resection for extrahepatic recurrent hepatocellular carcinoma. Hepatol Res 2020:50:978-984.

4. Hirokawa F, Hayashi M, Miyamoto Y, Asakuma M, Shimizu T, Komeda K, et al. Surgical treatment of extrahepatic recurrence of hepatocellular carcinoma. Langenbecks Arch Surg 2014;399:10571064.

5. Chon YE, Lee HA, Yoon JS, Park JY, Kim BH, Lee IJ, et al. Hepatocellular carcinoma in Korea between 2012 and 2014: an analysis of data from the Korean nationwide cancer registry. J Liver Cancer 2020;20:135-147.

6. Cheng AL, Kang YK, Chen Z, Tsao CJ, Qin S, Kim JS, et al. Efficacy and safety of sorafenib in patients in the Asia-Pacific region with advanced hepatocellular carcinoma: a phase III randomised, double-blind, placebo-controlled trial. Lancet Oncol 2009;10:25-34.

7. Ueshima K, Kudo M, Tanaka M, Kumada T, Chung H, Hagiwara S, et al. Phase I/II study of sorafenib in combination with hepatic arterial infusion chemotherapy using low-dose cisplatin and 5-fluorouracil. Liver Cancer 2015;4:263-273.

8. Kudo M, Ueshima K, Yokosuka O, Ogasawara S, Obi S, Izumi N, et al. Sorafenib plus low-dose cisplatin and fluorouracil hepatic arterial infusion chemotherapy versus sorafenib alone in patients with advanced hepatocellular carcinoma (SILIUS): a randomised, open label, phase 3 trial. Lancet Gastroenterol Hepatol 2018:3:424-432.

9. Ikeda M, Morizane C, Ueno M, Okusaka T, Ishii H, Furuse J. Chemotherapy for hepatocellular carcinoma: current status and future perspectives. Jpn J Clin Oncol 2018;48:103-114.

10. Ensminger WD, Rosowsky A, Raso V, Levin DC, Glode M, Come S, et al. A clinical-pharmacological evaluation of hepatic arterial infusions of 5-fluoro-2'-deoxyuridine and 5-fluorouracil. Cancer Res 1978;38(11 Pt 1):3784-3792.

11. Sinn DH, Cho JY, Gwak GY, Paik YH, Choi MS, Lee JH, et al. Different survival of Barcelona clinic liver cancer stage $C$ hepatocellular carcinoma patients by the extent of portal vein invasion and the type of extrahepatic spread. PLoS One 2015;10:e0124434.

12. Berger Y, Spivack JH, Heskel M, Aycart SN, Labow DM, Sarpel U. Extrahepatic metastasectomy for hepatocellular carcinoma: predictors of long-term survival. J Surg Oncol 2016;114:469-474.

13. Zetter BR. The cellular basis of site-specific tumor metastasis. $N$ Engl J Med 1990;322:605-612. 